\title{
Effects of Music Intervention during Cesarean Section on the Level of the Mother's Anxiety: A Randomized Controlled Study
}

\author{
Jule Eriç Horasanlı ${ }^{1}$ and NUR DEMIRBAS ${ }^{2}$ \\ ${ }^{1}$ Affiliation not available \\ ${ }^{2}$ Necmettin Erbakan Universitesi Meram Tip Fakultesi
}

April 5, 2021

\begin{abstract}
Background: Anxiety is commonly experienced during the delivery process and has been shown to have adverse effects on maternal and infant health outcomes. Music interventions tend to mitigate the effects of anxiety in a variety of populations, are low-cost and easily accessible, and have high acceptability. The aim of this study is to evaluate the effectiveness of music intervention on expectant mothers' vital signs and anxiety levels during cesarean section. Methods: This study is a single-center, controlled, randomized study. The women in the intervention group listened to music via earpieces during the operation. The control group received standard treatment without music. Cesarean section was performed under regional anesthesia. The State-Trait Anxiety Inventory (STAI) was administered to the patients before and after the operation. Results: There was no significant difference between the pre- and postoperative systolic blood pressure, diastolic blood pressure, heart rate, respiratory rate, or $\mathrm{O} 2$ saturation parameters of the music group and the control group $(\mathrm{p}>0.05)$. The pre-operative STAI anxiety score was similar in the music (45.58 \pm 4.60$)$ and control $(43.82 \pm 4.33)$ groups $(\mathrm{p}=0.183)$. After the operation, the anxiety score of the music group (35.88 \pm 5.39$)$ was found to be statistically significantly lower than the anxiety score of the control group (42.14 \pm 3.75 ) $(\mathrm{p}<0.001)$. Conclusion: This study supports the hypothesis that slow, rhythmic Suf music during cesarean section can reduce patients' anxiety. It is possible to distract the patient from negative thoughts and reduce stress. The results show that patients who listened to music during surgery had significantly lower anxiety levels than patients who did not listen to music.
\end{abstract}

\section{Effects of Music Intervention during Cesarean Section on the Level of the Mother's Anxiety: A Randomized Controlled Study}

\section{Abstract}

Background: Anxiety is commonly experienced during the delivery process and has been shown to have adverse effects on maternal and infant health outcomes. Music interventions tend to mitigate the effects of anxiety in a variety of populations, are low-cost and easily accessible, and have high acceptability. The aim of this study is to evaluate the effectiveness of music intervention on expectant mothers' vital signs and anxiety levels during cesarean section.

Methods: This study is a single-center, controlled, randomized study. The women in the intervention group listened to music via earpieces during the operation. The control group received standard treatment without music. Cesarean section was performed under regional anesthesia. The State-Trait Anxiety Inventory (STAI) was administered to the patients before and after the operation.

Results: There was no significant difference between the pre- and postoperative systolic blood pressure, diastolic blood pressure, heart rate, respiratory rate, or $\mathrm{O}_{2}$ saturation parameters of the music group and the control group $(\mathrm{p}>0.05)$. The pre-operative STAI anxiety score was similar in the music $(45.58 \pm 4.60)$ and control (43.82 \pm 4.33$)$ groups $(\mathrm{p}=0.183)$. After the operation, the anxiety score of the music group (35.88 \pm 5.39$)$ 
was found to be statistically significantly lower than the anxiety score of the control group $(42.14 \pm 3.75)$ $(\mathrm{p}<0.001)$.

Conclusion: This study supports the hypothesis that slow, rhythmic Sufi music during cesarean section can reduce patients' anxiety. It is possible to distract the patient from negative thoughts and reduce stress. The results show that patients who listened to music during surgery had significantly lower anxiety levels than patients who did not listen to music.

Keywords : Cesarean section, Anxiety, Sufi music

\section{What's known}

-Women undergoing cesarean delivery may have significant anxiety prior to surgery.

-Non-pharmacological approaches to anxiety reduction are favored in this patient population.

-Music interventions tend to mitigate the effects of anxiety in a variety of populations, are low-cost and easily accessible, and have high acceptability.

\section{What's new}

-The slow rhythmic music (Sufi music) can reduce the anxiety of patients during C-section.

-patients who listen to music during surgery have significantly lower anxiety levels than patients who do not listen to music.

- Music can be easily applied perioperatively by the assisting staff of the anesthesia or surgical team.

\section{INTRODUCTION}

The cesarean section (C-section) rate in Turkey is $51.2 \%^{1}$, well above the rate of $5-15 \%$ of deliveries suggested by the World Health Organization ${ }^{2}$. Births are a source of stress for women, and a C-section causes an increase in stress and anxiety for patients due to the fact that it is a major abdominal surgery and critical situations may occur ${ }^{3,4}$. High preoperative anxiety levels have been reported in many women who are prepared for a C-section ${ }^{5}$. Since it is not possible for the father or other supporter to attend a patient's C-section in Turkey, it can be expected that patients have higher anxiety levels due to isolation. There may also be potential negative side effects of premedication to deal with anxiety ${ }^{6}$. Due to the increased prevalence of C-sections, choosing non-pharmacological, non-invasive interventions for anxiety treatment would be advantageous for both the expectant mother and the baby.

Compared to general anesthesia, spinal anesthesia is a more preferred form of anesthesia because it reduces intraoperative blood loss and causes less nausea and vomiting, less respiratory depression, and less postoperative pain ${ }^{7}$. However, sounds originating from the surgical environment (white noise), such as the sounds of surgical instruments, are known to increase patient anxiety ${ }^{8}$. This situation occurs especially more frequently for pregnant women.

Music intervention as a stress management technique is an easy-to-apply, cost-effective, non-pharmacological, and non-invasive method that has been used for centuries ${ }^{9}$. While it positively supports the psychological well-being of the person, it may also improve physical parameters such as blood pressure, heart rate, and respiration ${ }^{10}$. It has been shown in some studies that listening to music increases the threshold of pain during an operation by distracting the patient's attention ${ }^{11}$. Music is effective in reducing pain by stimulating the autonomic nervous system and releasing endorphins and natural opioids from the pituitary gland ${ }^{12,13}$. It causes a decrease in blood pressure and heart rate by reducing catecholamines ${ }^{14}$. These effects generally vary depending on the tempo of the music; slow-paced and intermittent music has been associated with a decrease in heart rate, respiratory rate, and blood pressure, while fast-paced music has been associated with an increase in these parameters ${ }^{6}$. Music can have physiological effects on many areas of life and mental health. It can affect tension depending on the type, and it can also be used to create specific emotions ${ }^{15}$. The potential therapeutic effect of music has been attributed to its ability to reduce stress and alter arousal ${ }^{16}$. 
There are studies showing that Sufi music is widely used in the treatment of various psychiatric diseases ${ }^{17}$. Turkish music played on traditional Sufi instruments (combined with the sounds of water flowing from a fountain) was used throughout the times of both the Seljuk and Ottoman Empires, from the 11th to 18th century, to treat mental illnesses ${ }^{18}$. Sufis often engaged in music and treated psychological disorders with music. Turkish music types used in hospitals in Amasya, Sivas, Istanbul, and Edirne were classified and started to be used in treatments. In addition, Farabi Musiki-ul-kebir, the inventor of the oud instrument, studied music together with astronomy and associated it with psychology according to its effects on people ${ }^{19}$. However, there are currently limited studies on C-sections and music. This study aimed to compare patients receiving music therapy with a control group and to investigate the effects of Sufi music on anxiety scores and vital signs of patients undergoing C-sections for elective reasons.

During the study, we had two main goals:

1: To compare state anxiety scores before and after the operation in the music and control groups.

2: To determine whether music causes changes in patients' blood pressure, heart rate, respiratory rate, or partial $\mathrm{O}_{2}$ pressure level.

\section{METHODS}

Study Design: The research was conducted in accordance with the CONSORT guidelines between January 2021 and March 2021 as a single-center, controlled, randomized study.

Ethics, Consent, and Permissions: Ethical permission for the study was obtained from the NE University Ethical Committee (No. 2957, December 18, 2020).

Inclusion Criteria: Patients over 18 years of age with singleton term pregnancies (over 37 weeks) undergoing elective cesarean section (obstetric indications or patients with previous cesarean section) under regional anesthesia were included in the study.

Exclusion Criteria: Pregnant women with maternal hypertensive disorders (chronic hypertension or preeclampsia), insulin-dependent diabetes mellitus, intrauterine growth retardation, pregnancies at the 41st week, premature rupture of membranes, multiple pregnancies, in utero fetal death, known fetal anomalies, hearing impairment, or psychiatric disorders were excluded from the study.

Participants: Potential participating patients were enrolled in the study after being informed by a single researcher (JEH) about the purpose of the study and how data would be collected. Eligible participants were told that they would participate in a pain relief study during the operation. They were not informed about the role of music in order to conduct a single-blind test. After obtaining informed consent in writing and verbally, the following data were collected from all participants before the procedure: age, parity, previous cesarean section information, smoking history, and illnesses. Participants were randomized into an intervention group that would listen to music and a control group that would not listen to music. Randomization was done by the researchers using the coin-flip method, where tails represented the music group and heads represented the control group. Of the 60 pregnant women initially included in the study, five were excluded from the study because they were switched to general anesthesia during the operation, three decided not to participate in the study, and three did not complete the questionnaire completely (Figure 1).

\section{Intervention}

The music to be played during the C-section was chosen as a 28-minute piece of Sufi music with a slow rhythm (ranging from 60 to 72 beats per minute as measured by a metronome), with a low to medium tone and a harmonious melody (https://www.youtube.com/watch? $v=h 374 R P f U o X 0) .28$ ). When the pregnant woman was taken to the intervention table before the C-section, the music was begun via an earpiece before the spinal anesthesia. During the spinal anesthesia, commands were communicated to the patient at a low decibel level. Unnecessary staff conversations were prevented to avoid white noise. Apart from that, the patient was allowed to communicate with hospital staff. The music continued to play throughout the operation. The control group heard the normal ambient white noise without any music intervention. 
Two interviews were conducted with these patients before and after the C-section. Fifteen minutes before admission to the operating room, the participant's information form containing sociodemographic and obstetric characteristics and the State-Trait Anxiety Inventory (STAI) questionnaire were administered face-to-face by the researcher. During and after the operation, heart rate, blood pressure, respiratory rate, and oxygen saturation were followed and recorded. After the operation, the STAI-1 scale was re-applied by the researcher at the 30th minute in the post-anesthesia care unit.

\section{Instruments}

State-Trait Anxiety Inventory (STAI): The STAI, developed by Spielberger et al., consists of two parts with 20 items: the STAI-1, which measures the state anxiety level, and the STAI-2, which measures the trait anxiety level ${ }^{20}$. The scale is a four-point Likert-type scale with possible answers of "not at all," "a little," "a lot," and "completely." The STAI contains two types of statements. Positive (direct) expressions express negative emotions, while negative (inverted) expressions express positive emotions. Negative statements in the STAI-1 (state anxiety) are items 1, 2, 5, 8, 10, 11, 15, 16, 19, and 20. Negative statements in the STAI-2 (trait anxiety) are items 21, 26, 27, 30,33, 36, and 39. Separate total weights of direct and inverted expressions are calculated and the sum of inverse expressions is subtracted directly from the sum of expressions. A predetermined and unchanging value is added to that number. This constant value is 50 for the STAI-1 and 35 for the STAI-2. The last value obtained is the anxiety score of the individual. If more than 3 statements are not answered from among the scale's 20 items, the form is considered invalid. The scores obtained from both scales theoretically vary between 20 and 80, where a high score indicates a high level of anxiety.

\section{Vital Signs}

Vital signs (include systolic and diastolic blood pressure, heart rate, and respiratory rate) were monitored by the researcher.

\section{Statistical Analysis}

The SPSS 24 statistical software package (IBM Corp., Armonk, NY, USA) was used for all data analysis. All data obtained from the questionnaires filled out during face-to-face interviews were evaluated and the means and standard deviations of the numerical data were calculated. Conformity to normal distribution was evaluated with the Kolmogorov-Smirnov test, and categorical and numerical data were compared with appropriate statistical analyses such as the chi-square test, independent t-test, and one-way ANOVA. Values of $\mathrm{p}<0.05$ were considered statistically significant. Reliability scores of each scale were calculated with Cronbach's alpha, and pre- and postoperative data were compared with paired sample t-tests between the case and control groups.

\section{RESULTS}

A total of 49 pregnant women, with 26 in the music intervention group and 23 in the control group, were included in this study. The mean age of the women included in the music group was $31.25 \pm 5.81$ years while the mean age of the women included in the control group was $29.64 \pm 5.97$ years. There was no statistical difference between the demographic variables of the music group and the control group ( $\mathrm{p}>0.05$ ) (Table 1).

There was no significant difference between the preoperative systolic blood pressure, diastolic blood pressure, heart rate, respiratory rate, and $\mathrm{O}_{2}$ saturation parameters of the music group and the control group ( $\left.\mathrm{p}>0.05\right)$. After the operation, systolic blood pressure, diastolic blood pressure, heart rate, respiratory rate, and $\mathrm{O}_{2}$ saturation parameters were similar between the groups $(\mathrm{p}>0.05)$.

According to the STAI-1 scale applied to the pregnant woman before the operation, the anxiety score of the music group was $45.58 \pm 4.60$ and that of the control group was $43.82 \pm 4.33$; there was no statistically significant difference between the groups $(\mathrm{p}=0.183)$. After the operation, the anxiety score of the music group (35.88 \pm 5.39$)$ was found to be statistically significantly lower than the anxiety score of the control group $(42.14 \pm 3.75)(\mathrm{p}<0.001)$. 
Pre- and postoperative systolic blood pressure, diastolic blood pressure, and oxygen saturation were found to be unchanged in the music group. The heart rate and respiratory rate of the women were found to be significantly lower postoperatively than before the operation $(\mathrm{p}<0.001)$. In the control group, vital parameters did not change before and after the operation $(\mathrm{p}>0.05)$.

In the music group, the anxiety score before the operation was $45.58 \pm 4.60$, while the postoperative anxiety score was $35.88 \pm 5.39$. The postoperative anxiety score was found to be statistically significantly lower $(\mathrm{p}<0.001)$. In the control group, there was no significant change in anxiety levels before and after the operation $(\mathrm{p}=0.071)$.

\section{DISCUSSION}

The presented study is one of the few randomized controlled studies investigating the effect of music on anxiety during C-section in Turkey. In this study, it was observed that the postoperative anxiety scores of pregnant women who underwent C-sections while listening to Sufi music were significantly lower than those of the control group. In the music group, the postoperative anxiety score was also lower than the preoperative anxiety score.

It is generally known that waiting for and undergoing surgical procedures can be stressful situations. Patients who undergo surgical procedures usually experience anxiety because of the fear of experiencing complications ${ }^{21}$. Many anesthesiologists prefer epidural or spinal anesthesia during C-sections. Especially during uterine manipulation or the birth of the baby, the mother feels some discomfort. It is important for the expectant mother to be comfortable and cooperative in a C-section delivery to be performed with regional anesthesia. With music used as a relaxation tool, the mother may be better able to participate in the events of the surgical procedure ${ }^{22}$. Relaxing features of music include a fluent lyrical melody, simple harmony, and easy rhythm with soft tone (about $60-80$ beats/min). Music can stimulate the release of endorphins from the brain, lowering ACTH levels in the blood. Nerve impulses triggered by calming music can trigger muscle tone, brainwave frequency, galvanic skin response, pilomotor reflexes, and autonomic nervous system reactions ${ }^{21}$.

In a previous study, it was shown that there was a significant decrease in systolic blood pressure in the music group, which listened to a 40-minute music recording before undergoing C-section, while diastolic blood pressure and respiratory rate increased in the control group ${ }^{22}$. It has been shown that heart rate, systolic and diastolic blood pressure, and the need for sedation are significantly reduced in patients who listen to music recordings during colonoscopy procedures. Additionally, in both of these previous studies, anxiety levels were found to be lower in the groups listening to music ${ }^{23}$. In the present study, however, music was played for the patients intraoperatively, and no difference was observed between heart rate, systolic and diastolic blood pressure, respiratory rate, or partial $\mathrm{O}_{2}$ pressure between the groups. The reason for this is that uterotonic drugs (oxytocin) administered during spinal anesthesia and postpartum cause hypotension, reflex tachycardia, and respiratory changes, which may have affected the accuracy of the analysis.

The effect of listening to music on patient anxiety before and during surgery has been the subject of various studies. There are some contradictions in the literature regarding the effectiveness of listening to music on patients' anxiety during surgery. Some authors report that music is effective in reducing anxiety before and during surgery ${ }^{22,24}$. Music has been used to reduce stress, anxiety, and depression in pregnant women. In a metanalysis, it was suggested that music has a positive and statistically significant effect in reducing the psychological parameters of state anxiety before surgical procedures ${ }^{25}$. In another systematic review, it was concluded that music played with headphones reduced the anxiety of patients during normal labor ${ }^{26}$. However, there are also studies stating that listening to music does not affect anxiety and pain outcomes ${ }^{26,27}$. In the present study, no difference was observed between the music group's mean anxiety score and that of the control group according to the STAI-1 questionnaire applied to the pregnant woman before the operation. This finding may be due to the intraoperative application of the music in this study. After the operation, the anxiety score of the music group was found to be statistically significantly lower than the anxiety score of the control group. Additionally, in the music group, the postoperative anxiety score was found to be lower than the preoperative anxiety score. In other words, listening to music reduced the anxiety scores of the patients 
in the intervention group.

It is not clearly known whether a specific type of music has a superior effect on anxiety ${ }^{27}$. There are studies showing that the effect of Sufi music on anxiety is insufficient to create a significant difference in state and trait anxiety ${ }^{28,29}$. However, there are also studies showing the effect of Sufi music in reducing state anxiety during surgical procedures ${ }^{30,31}$. There is evidence that Sufi music therapy has a positive effect on state anxiety. This suggests that Suf music therapy may be useful in reducing anxiety in certain situations such as surgery or medical procedures. In a metaanalysis, Gürbüz et al. concluded that Sufi music may have a more beneficial effect when played once rather than repeatedly during a medical or surgical procedure ${ }^{30}$. In the present study, in accordance with these results, music was applied only during the operation. The positive effect of intraoperative music has been seen in our study, but it cannot be said whether this effect is specific to Suf music, since we did not apply and compare other types of music. Therefore, new comparative research is needed to evaluate the impact of Sufi music on state anxiety during C-section compared to other music genres. Sufi music was chosen in this study because it is a relaxing type of music.

Conclusion: This study supports the hypothesis that slow rhythmic music (Sufi music) can reduce the anxiety of patients during C-section. It is possible to distract the patient from negative thoughts and reduce stress. The study results show that patients who listen to music during surgery have significantly lower anxiety levels than patients who do not listen to music. It was determined that music performance did not cause changes in heart rate and blood pressure or partial $\mathrm{O}_{2}$ pressure level. As a result, music appears to be beneficial to aid in patient relaxation during cesarean delivery. We think that it can be easily applied perioperatively by the assisting staff of the anesthesia or surgical team.

Limitations of the study: An important limitation of this study was the process of determining how anxious participants felt during the surgical procedure with the postoperative questionnaire. Participants may not have been able to report exactly how anxious they felt during surgery, already experiencing relief with the birth of the baby. Another important factor that may have affected the results is that although the term "anxiety" does not appear in the STAI scale, some questions posed may have caused patients to predict the hypothesis of a research study on anxiety and change their behavior. The researcher's presence throughout the procedure may also have contributed to the participants having a more positive response.

\section{References}

1. Flamm BL. Cesarean section: a worldwide epidemic? Birth (Berkeley, Calif). 2000;27(2):139-40.

2. Eyı EGY, Mollamahmutoglu L. An analysis of the high cesarean section rates in Turkey by Robson classification. The Journal of Maternal-Fetal \& Neonatal Medicine. 2019:1-11.

3. Lipson JG. Repeat Cesarean Births Social and Psychological Issues. Journal of Obstetric, Gynecologic, \& Neonatal Nursing. 1984;13(3):157-62.

4. Allen VM, O'Connell CM, Liston RM, Baskett TF. Maternal morbidity associated with cesarean delivery without labor compared with spontaneous onset of labor at term. Obstetrics \& Gynecology. 2003;102(3):477-82.

5. Wyatt S, Jones D, Peach M, Gurrin L. Anxiety in patients having caesarean section under regional anaesthesia: a questionnaire and pilot study. International journal of obstetric anesthesia. 2001;10(4):278-83.

6. Bernardi L, Porta C, Casucci G, Balsamo R, Bernardi NF, Fogari R, et al. Clinical Perspective. Circulation. 2009;119(25):3171-80.

7. Barash P, Cullen B, Stoelting R, Cahalan M, Stock M. Clinical anesthesia. Philadelphia: Lippicott Williams \& Wilkins. 2006:691-717.

8. Lepage C, Drolet P, Girard M, Grenier Y, DeGagné R. Music decreases sedative requirements during spinal anesthesia. Anesthesia \& Analgesia. 2001;93(4):912-6.

9. Wang Y, Dong Y, Li Y. Perioperative psychological and music interventions in elderly patients undergoing spinal anesthesia: effect on anxiety, heart rate variability, and postoperative pain. Yonsei medical journal. 2014;55(4):1101.

10. Lane D, editor Music therapy: A gift beyond measure. Oncology Nursing Forum; 2006: Oncology 
Nursing Society.

11. Stevens K. Patients' perceptions of music during surgery. Journal of advanced nursing. 1990;15(9):104551.

12. Cook JD. Music as an intervention in the oncology setting. Cancer Nursing. 1986;9(1):23-8.

13. Beck S, editor The therapeutic use of music for cancer-related pain. Oncology nursing forum; 1991.

14. Coughlan A. Music therapy in ICU. Nursing times. 1994;90(17):35-.

15. McCraty R, Barrios-Choplin B, Atkinson M, Tomasino D. The effects of different types of music on mood, tension, and mental clarity. Alternative therapies in health and medicine. 1998;4(1):75-84.

16. Chanda ML, Levitin DJ. The neurochemistry of music. Trends in cognitive sciences. 2013;17(4):179-93.

17. Anatolia AM. An example for the application of music therapy in the medical history: Divrigi Darüssifa. Acta Med Anatol. 2014;3(2):63-6.

18. Wigram, T.; Saperston, B.; West, R. (Eds.) The Art and Science of Music Therapy: A Handbook, The Netherlands: Harwood Academic Publishers. 2009

19. Erdal G, Erbaş I. Darussifas Where Music Threapy was Practiced During Anatolian Seljuks and Ottomans. Journal of History Culture and Art Research. 2013;2(1):1-19.

20. Spielberger CD. Manual for the State-trait Anxietry, Inventory. Consulting Psychologist. 1970.

21. Kaminski J, Hall W. The effect of soothing music on neonatal behavioral states in the hospital newborn nursery. Neonatal network : NN. 1996;15(1):45-54. Epub 1996/02/01.

22. Kushnir J, Friedman A, Ehrenfeld M, Kushnir T. Coping with preoperative anxiety in cesarean section: physiological, cognitive, and emotional effects of listening to favorite music. Birth. 2012;39(2):121-7.

23. Smolen D, Topp R, Singer L. The effect of self-selected music during colonoscopy on anxiety, heart rate, and blood pressure. Applied Nursing Research. 2002;15(3):126-36.

24. Voss JA, Good M, Yates B, Baun MM, Thompson A, Hertzog M. Sedative music reduces anxiety and pain during chair rest after open-heart surgery. Pain. 2004;112(1-2):197-203.

25. Evans D. The effectiveness of music as an intervention for hospital patients: a systematic review. Journal of advanced nursing. 2002;37(1):8-18.

26. Good M. A comparison of the effects of jaw relaxation and music on postoperative pain. Nursing research. 1995.

27. Colt HG, Powers A, Shanks TG. Effect of music on state anxiety scores in patients undergoing fiberoptic bronchoscopy. Chest. 1999;116(3):819-24.

28. Ozdemir U, Tascı S, Yıldızhan E, Aslan S, Eser B. The effect of classical turkish music on pain severity and anxiety levels in patients undergoing bone marrow aspiration and biopsy. Pain Management Nursing. 2019;20(1):82-7.

29. Kocabas P, Khorshid L. A comparison of the effects of a special gynaecological garment and music in reducing the anxiety related to gynaecological examination. Journal of Clinical Nursing. 2012;21(56):791-9.

30. Gurbuz-Dogan R, Ali A, Candy B, King M. The effectiveness of Sufi music for mental health outcomes. A systematic review and meta-analysis of 21 randomised trials. Complementary Therapies in Medicine. 2021:102664.

\section{Hosted file}

tables.pdf available at https://authorea.com/users/405821/articles/516668-effects-of-musicintervention-during-cesarean-section-on-the-level-of-the-mother-s-anxiety-a-randomizedcontrolled-study

\section{Hosted file}

flow-chart.pdf available at https://authorea.com/users/405821/articles/516668-effectsof-music-intervention-during-cesarean-section-on-the-level-of-the-mother-s-anxiety-arandomized-controlled-study

\section{Hosted file}


figure 2.pdf available at https://authorea.com/users/405821/articles/516668-effects-ofmusic-intervention-during-cesarean-section-on-the-level-of-the-mother-s-anxiety-arandomized-controlled-study 